\title{
Contribución a la determinación de los contenidos de yeso y hemihidrato en los cementos portland
}

\author{
FERNANDO MORENO ARUS Y IULIO PEREZ ALONSO \\ Laboratorio Central de Eisayos de la Compañía General de Asfaltos Y Portiand Asiand
}

\section{INTRODUCCION}

La mayoría de los técnicos de cemento eceptan que las anormalidades del fraguado, conocidas como "falso fraguado" en el cemento portland, se deben primordialmente a la presencia de yeso parcialmente deshidratado $\left(\mathrm{SO}_{4} \mathrm{Ca} \quad{ }^{1 / 2} \mathrm{H}_{2} \mathrm{O}\right)$. Si el clínker que se muele está enriquecido en cal libre, o la temperatura del molino es elevada (superior a los $110^{\circ} \mathrm{C}$ ) o hay escasa ventilación de éste, se llega a originar una parcial deshidratación del yeso, que se mantiene durante el proceso de ensilado y origina las anormalidades del fraguado anteriormente referidas. Por esta razón creemos muy importante el poder conocer el grado de deshidratación en que se encuentra el yeso en un cemento.

Son varios los autores que han basado la identificación de cada una de las formas del sulfato cálcico presentes en el cemento molido, por las áreas de los picos endotérmicos de los termogramas obtenidos por análisis térmico diferencial.

K. Mura-Kamil y colaboradores 1957 (1), K. Takemoto e Ik Hirayama 1959 (2), y J. Dyczck y J. Jerzy Kawkow 1963 (3) iniciaron una estimación semi-cuantitativa de la proporción relativa de yeso y hemihidrato presentes en el cemento, partiendo de mezclaspatrón de yeso y hemihidrato.

Basándonos en el mismo tipo de mezclas-patrón, J. Sulikowski 1965 (4) y R. A. Kuntze (5) determinan, de una forma cuantitativa, las proporciones de hidratos del yeso, logrando especialmente éste último una mejora en el método al conseguir retardar el segundo efecto endotérmico de la pérdida de la $1 / 2$ molécula de agua de cristalización restante, realizando el ATD en atmósfera saturada de vapor de agua a $760 \mathrm{~mm} \mathrm{Hg}$. Con ello consigue una separación clara de los dos picos endotérmicos que facilita la determinación de sus áreas.

N. Sato y M. Kanaya (7), siguiendo la técnica de mezclas-patrones de clínker, yeso y hemihidrato, efectuaron un estudio análogo a los anteriores, profundizando sobre la influencia de la granulometría de las muestras y la naturaleza del yeso empleado, encontrando que la finura de las muestras no efecta, de manera importante, en el intervalo entre 3.000 y $5.000 \mathrm{~cm}^{2} / \mathrm{g}$ (Blaine), y el tipo de yeso sólo, en algún caso muy particular, puede afectar a las áreas de los picos endotérmicos de la deshidratación del yeso y hemihidrato. 
Asimismo, D. A. S. John (8) hace hincapié en que las rectas de tarado de las mezclas de yeso, hemihidrato y clínker varían según el método seguido para la preparación del hemihidrato; indicando que el método correcto es el de provocar una deshidratación parcial del yeso mezclado con clínker mediante la molienda a mano, con lo cual obtiene una mezcla de clínker, yeso y hemihidrato producido por el calor de la molienda. La riqueza en hemihidrato viene determinada por la pérdida al fuego a $215^{\circ}-230^{\circ} \mathrm{C}$, corrigiendo finalmente con yeso puro para obtener las diferentes proporciones de yeso y hemihidrato que constituyen los patrones.

Nosotros hemos intentado aportar al método de las áreas del ATD, utilizado por los autores anteriormente citados, dos aspectos nuevos:

Uno de ellos es el efectuar una concentración previa de las muestras mediante eliminación por ataque con metanol ácido salicílico de los silicatos. Dicha concentración da lugar a una mayor diferenciación de los efectos endotérmicos e incrementa sus intensidades por enriquecimiento de las dos fases hidratadas del yeso.

Otro aspecto es el empleo del análisis térmico diferencial doble, que por su fundamento elimina la influencia de la naturaleza del propio yeso (7) y de sus correspondientes fases deshidratadas (8) (citadas por Sato, Kanaya y John) y, sobre todo, los errores procedentes de las derivadas del análisis instrumental, que se atenúan al efectuar simultáneamente el análisis de la muestra-problema y la patrón.

\section{PARTE EXPERIMENTAL}

\subsection{Preparación de los cementos}

Los materiales de partida son: clínker molido a una superficie específica Blaine de 5.900 $\mathrm{cm}^{2} / \mathrm{g}$, yeso mineral y hemihidrato, molidos ambos a una finura menor de 60 micras. El hemihidrato ha sido preparado a partir de yeso mineral, molido a la finura indicada y tratado a $130^{\circ} \mathrm{C}$ en una estufa de desecación, controlando la pérdida correcta de la molécula y media de agua, con el análisis térmico diferencial (ATD) y difracción de rayos $\mathrm{X}$ (DRX).

En las muestras de clínker, yeso y hemihidrato, preparadas según lo anteriormente descrito, se determinó su contenido en $\mathrm{SO}_{3}$, resultando ser $0,82,40,48$ y $45,50 \%$, respectivamente.

Partiendo de estas tres materias se prepararon tres grupos de cementos (serie A) con 4,50, 3,50 y $2,75 \%$ de $\mathrm{SO}_{3}$.

A continuación exponemos las diferentes composiciones de los cementos con 4,50, 3,50 y $2,75 \%$ de $\mathrm{SO}_{3}$ :

Cementos: grupo Ia, Serie A 4,50\% $\mathrm{SO}_{3}$, total previsto

\begin{tabular}{|c|c|c|c|c|c|}
\hline$\%$ clinker & $\%$ yeso & $\%$ hemihidrato & $\begin{array}{c}\% \text { yeso en la } \\
\text { adición }\end{array}$ & Denominación & $\begin{array}{l}\mathrm{SO}_{3} \text { total } \\
\text { determinado }\end{array}$ \\
\hline 90,7 & 9,3 & - & 100 & $\mathrm{Y}$ & 4,60 \\
\hline 91,3 & 5,8 & 2,9 & 66,66 & $\frac{\mathrm{Y}}{\mathrm{H}}=\frac{2}{1}$ & 4,40 \\
\hline 91,4 & 4,3 & 4,3 & 50 & $\frac{\mathrm{Y}}{\mathrm{H}}=\frac{1}{1}$ & 4,50 \\
\hline 91,6 & 2,8 & 5,6 & 33,33 & $\frac{\mathrm{Y}}{\mathrm{H}}=\frac{1}{2}$ & 4,30 \\
\hline 91,8 & - & 8,2 & 0 & $\mathrm{H}$ & 4,40 \\
\hline
\end{tabular}


Cementos: grupo $\mathrm{Ib}$, Serie $\mathrm{B} 4,50 \% \mathrm{SO}_{3}$, total previsto

\begin{tabular}{cccccc}
\hline clínker & $\%$ yeso & $\%$ hemihidrato & $\begin{array}{c}\text { \% yeso en la } \\
\text { adición }\end{array}$ & Denominación & $\begin{array}{c}\text { so }_{3} \text { total } \\
\text { determinado }\end{array}$ \\
\hline 90,7 & 9,3 & - & 100 & $\mathrm{Y}$ & 4,32 \\
91,3 & 5,8 & 2,9 & 66,66 & $\frac{\mathrm{Y}}{\mathrm{H}}=\frac{2}{1}$ & 4,38 \\
91,4 & 4,3 & 4,3 & 50 & $\frac{\mathrm{Y}}{\mathrm{H}}=\frac{1}{1}$ & 4,54 \\
91,6 & 2,8 & 5,6 & 33,33 & $\frac{\mathrm{Y}}{\mathrm{H}}=\frac{1}{2}$ & 4,51 \\
91,8 & - & 8,2 & 0 & $\mathrm{H}$ & 4,70 \\
\hline
\end{tabular}

Cementos: grupo IIa, Serie A $3,50 \% \mathrm{SO}_{3}$, total previsto

\begin{tabular}{ccccccc}
\hline clinker & $\%$ yeso & $\%$ hemihidrato & $\begin{array}{c}\% \text { yeso en la } \\
\text { adición }\end{array}$ & Denominación & $\begin{array}{c}\text { SO }_{3} \text { total } \\
\text { determinado }\end{array}$ \\
\hline 93,2 & 6,8 & - & 100 & $\mathrm{Y}$ & 3,32 \\
93,4 & 4,4 & 2,2 & 66,66 & $\frac{\mathrm{Y}}{\mathrm{H}}=\frac{2}{1}$ & 3,54 \\
93,6 & 3,2 & 3,2 & 50 & $\frac{\mathrm{Y}}{\mathrm{H}}=\frac{1}{1}$ & 3,62 \\
93,7 & 2,1 & 4,2 & 33,33 & $\frac{\mathrm{Y}}{\mathrm{H}}=\frac{1}{2}$ & 3,64 \\
94,0 & - & 6,0 & 0 & $\mathrm{H}$ & 3,64 \\
\hline
\end{tabular}

Cementos: Grupo IIIa, Serie A $2,75 \% \mathrm{SO}_{3}$, total previsto

\begin{tabular}{cccccc}
\hline$\%$ clinker & $\%$ yeso & $\%$ hemihidrato & $\begin{array}{c}\% \text { yeso en la } \\
\text { adición }\end{array}$ & Denominación & $\begin{array}{c}\text { SO }_{3} \text { total } \\
\text { determinado }\end{array}$ \\
\hline 95,1 & 4,9 & - & 100 & $\mathrm{Y}$ & 2,56 \\
95,2 & 3,2 & 1,6 & 66,66 & $\frac{\mathrm{Y}}{\mathrm{H}}=\frac{2}{1}$ & 2,74 \\
95,4 & 2,3 & 2,3 & 50 & $\frac{\mathrm{Y}}{\mathrm{H}}=\frac{1}{1}$ & 2,60 \\
95,5 & 1,5 & 3,0 & 33,33 & $\frac{\mathrm{Y}}{\mathrm{H}}=\frac{1}{2}$ & 2,88 \\
95,7 & - & 4,3 & 0 & $\mathrm{H}$ & 2,60 \\
\hline
\end{tabular}

Cada grupo consta de cinco muestras obtenidas mezclando en diferentes proporciones los tres componentes, de tal forma, que el $\mathrm{SO}_{3}$ total sea constante en cada grupo, y la relación yeso-hemihidrato varíe. La composición correcta de las muestras se comprobó mediante la determinación del $\mathrm{SO}_{3}$ total. 
Para conocer la reproductividad de los ensayos se preparó otro grupo de cementos (Serie B) con idénticas dosificaciones, utilizando el mismo clínker y yeso de igual procedencia y riqueza.

De las dos series de cementos se preparan también muestras "concentradas", por eliminación de la mayor parte del clínker (el $80 \%$ ) por ataque de $10 \mathrm{~g}$ de muestra con $70 \mathrm{~g}$ de ácido salicílico y $400 \mathrm{ml}$ de metanol durante una hora, filtración y secado a $65^{\circ} \mathrm{C}$ hasta peso constante.

\subsection{Análisis Térmico Diferencial}

Los termogramas se efectuaron con un equipo Nestzch, con las siguientes condiciones:

\begin{tabular}{|c|c|}
\hline Velocidad de calentamiento & 2 grados $/ \mathrm{min}$ \\
\hline Sensibilidad & $\pm 0,05 \mathrm{mV}$ \\
\hline Muestra de referencia & $\mathrm{Al}_{2} \mathrm{O}_{3}-\gamma$ \\
\hline $\begin{array}{llllll}\text { Termopares } & \ldots & \ldots & \ldots & \ldots\end{array}$ & Pt-Pt $10 \% \mathrm{Rh}$ \\
\hline Velocidad papel del registro & $120 \mathrm{~mm} /$ hora \\
\hline Peso muestra & $0,6 \mathrm{~g}$ \\
\hline
\end{tabular}

En las dos series de cementos analizadas sus termogramas (fig. 1) presentan dos reacciones endotérmicas: una a $130^{\circ} \mathrm{C}$ correspondiente al paso $\mathrm{Ca} \cdot \mathrm{SO}_{4} \cdot 2 \mathrm{H}_{2} \rightarrow \mathrm{CaSO}_{4} \cdot 1 / 2 \mathrm{H}_{2} \mathrm{O}$ y otra a $140^{\circ}-145^{\circ} \mathrm{C}$ de la transformación Ca. $\mathrm{SO}_{4} \cdot 1 / 2 \mathrm{H}_{2} \mathrm{O} \rightarrow \mathrm{CaSO}_{4}$ anhidrita soluble. El hemihidrato presente a $140^{\circ}-145^{\circ} \mathrm{C}$ puede proceder del que entra a formar parte de la muestra inicial, y/o del formado à partir del yeso en la deshidratación parcial a $130^{\circ} \mathrm{C}$.

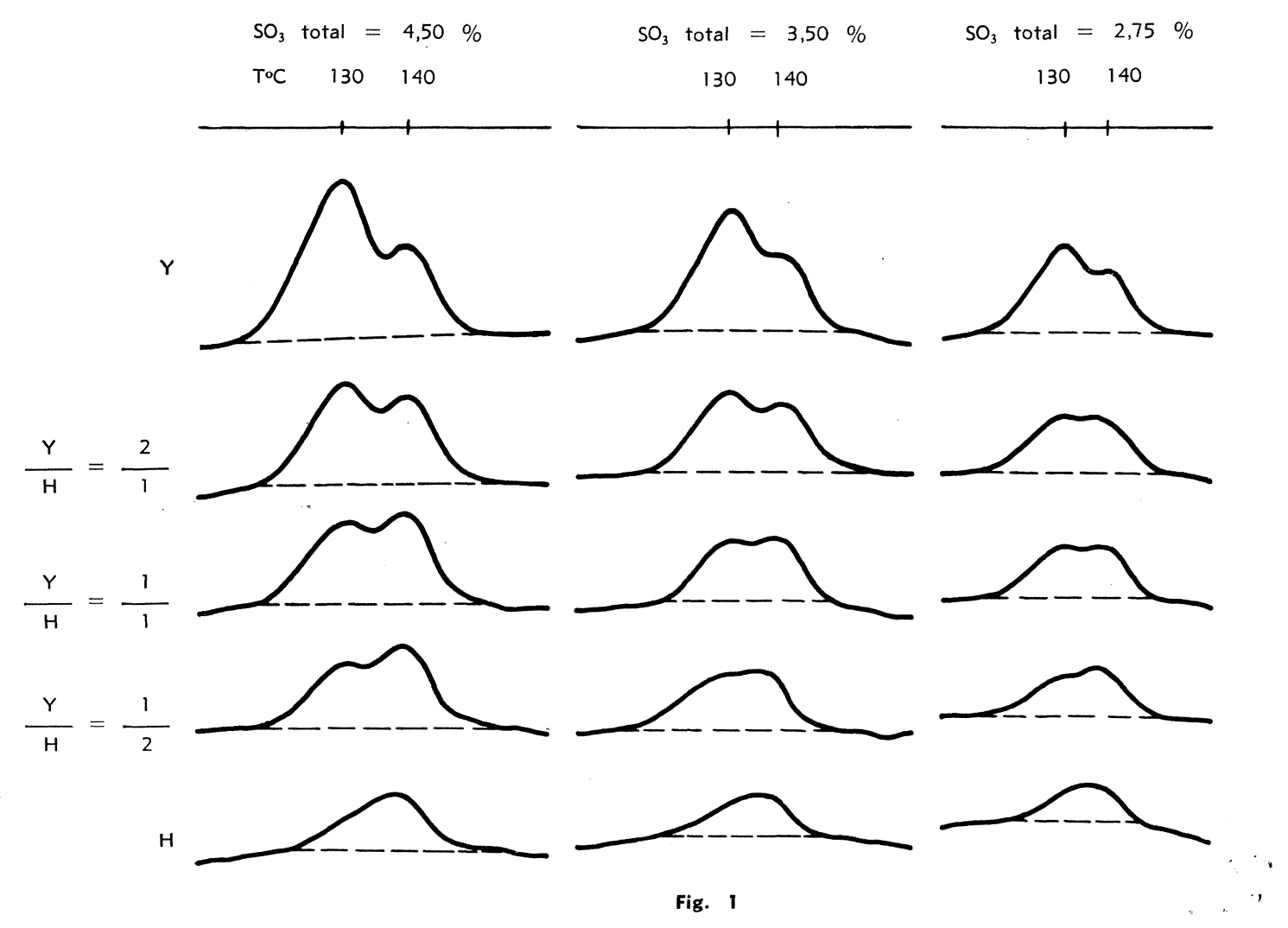


Por lo tanto, para cementos con el mismo contenido en $\mathrm{SO}_{3}$, a medida que se sustituye el yeso por hemihidrato, el pico a $130^{\circ} \mathrm{C}$, correspondiente a la pérdida de una y media moléculas de agua de hidratación, disminuye hasta desaparecer en las muestras en que todo el yeso se ha sustituido por hemihidrato.

Paralelamente se observa una lenta disminución del pico a $140^{\circ}-145^{\circ} \mathrm{C}$, que debe ser la resultante de dos efectos opuestos: uno positivo, determinado por el mayor contenido de agua presente a $140^{\circ}-145^{\circ} \mathrm{C}$ y consecuencia de la sustitución del yeso por hemihidrato, y otro negativo, por la dilución (aumento de la proporción del clínker con respecto al yeso-hemihidrato) que implica la sustitución del yeso por el hemihidrato, más rico en $\mathrm{SO}_{3}$, al mantener el $\mathrm{SO}_{3}$ constante.

Las alturas de los dos picos endotérmicos en relación con el contenido de yeso, para cementos con igual contenido en $\mathrm{SO}_{3}$, vienen en la tabla 1 .

TA B L A 1

\begin{tabular}{|c|c|c|c|c|c|c|}
\hline \multirow{2}{*}{$\%$ yeso } & \multicolumn{2}{|c|}{$4,50 \% \mathrm{SO}_{3}$ total } & \multicolumn{2}{|c|}{$3,50 \% \mathrm{SO}_{3}$ total } & \multicolumn{2}{|c|}{$2,75 \% \mathrm{SO}_{3}$ total } \\
\hline & $h_{1}$ & $h_{2}$ & $h_{1}$ & $h_{2}$ & $\mathbf{h}_{1}$ & $h_{2}$ \\
\hline 100 & 41 & 24 & 31 & 20 & 22 & 16 \\
\hline 66,6 & 27 & 24 & 21 & 18 & 15 & 14 \\
\hline 50 & 21 & 23 & 16 & 16 & 14 & 14 \\
\hline 33,3 & 17 & 21 & 14 & 15 & 10 & 13 \\
\hline 0 & 0 & 15 & 0 & 11 & 0 & 9 \\
\hline $\mathrm{R}_{1}$ & 0,995 & 0,887 & 0,991 & 0,989 & 0,983 & 0,950 \\
\hline
\end{tabular}

$$
\begin{aligned}
& h_{1}=\text { Altura en } \mathrm{mm} \text { pico a } 130^{\circ} \mathrm{C} . \\
& h_{2}=\text { Altura en mm pico a } 140^{\circ}-145^{\circ} \mathrm{C} . \\
& R_{1}=\text { Coeficiente de correlación correspondiente al \% de yeso con cada una de las variables consideradas. }
\end{aligned}
$$

Estas alturas para los diferentes contenidos de yeso se representaron en las gráficas I, II y III.

Los termogramas de las muestras "concentradas" por ataque tienen un aspecto más interesante (fig. 2). Poseen una separación mayor, entre los dos picos característicos, presentándose el segundo pico a $150^{\circ}-155^{\circ} \mathrm{C}$, incrementando, por lo tanto, la diferencia de temperaturas entre ambos picos a $25^{\circ} \mathrm{C}$. En estas muestras concentradas la altura de los picos es del orden del doble de las correspondientes a las muestras normales, pasando de 4 a 10 $\mathrm{mm}$, por cada $1 \%$ de yeso presente en la muestra inicial.

Por lo cual es posible determinar, además de las alturas de los picos, el área correspondiente mediante planímetro, con la ayuda de unas líneas auxiliares (tabla 2).

Los valores de las alturas y áreas en función del porcentaje de yeso están representados en las gráficas IVA, IVB, V, VI y VII. 


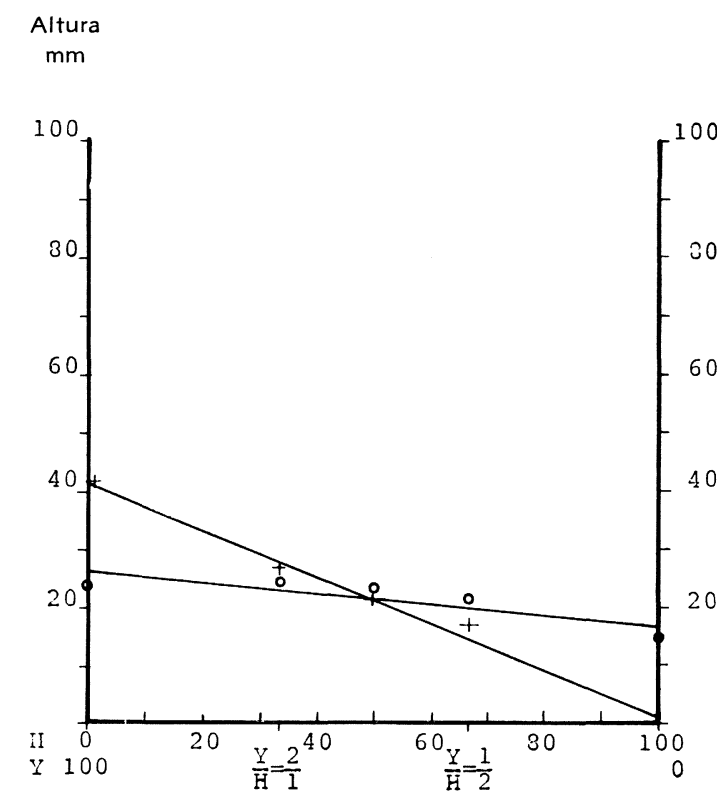

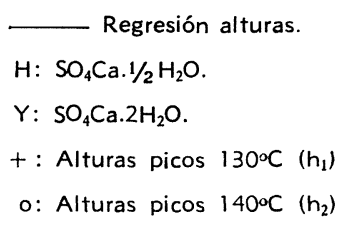

- Regresión alturas.

$\mathrm{H}: \mathrm{SO}_{4} \mathrm{Ca} \cdot 1 / 2 \mathrm{H}_{2} \mathrm{O}$.

$\mathrm{Y}: \mathrm{SO}_{4} \mathrm{Ca} .2 \mathrm{H}_{2} \mathrm{O}$.

+ : Alturas picos $130^{\circ} \mathrm{C}\left(h_{1}\right)$

o: Alturas picos $140^{\circ} \mathrm{C}\left(h_{2}\right)$

$\mathrm{SO}_{3}=4,50$ Serie $\mathrm{A}$.

P'eso muestra 0,6 g.

Gráfica I

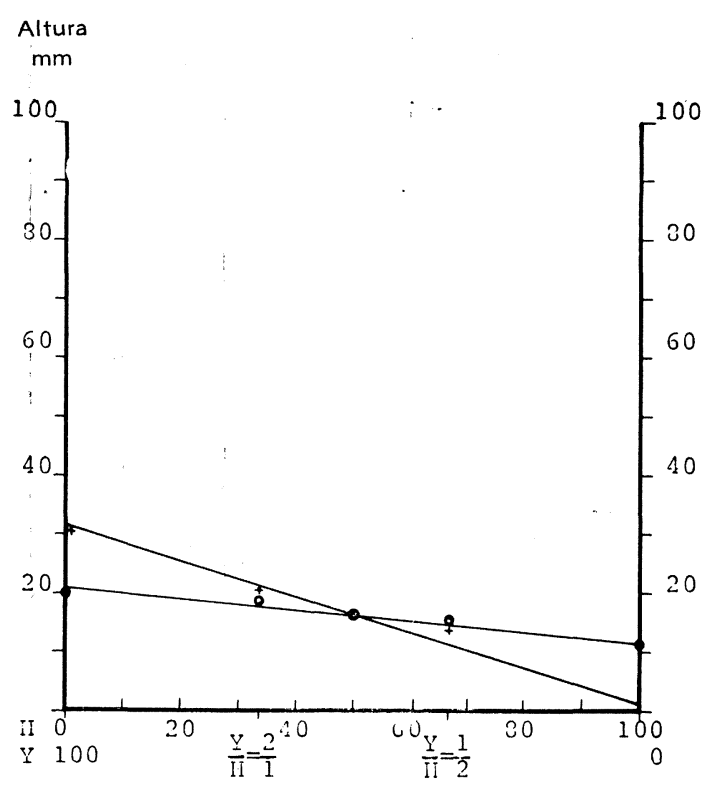

Altura

$\mathrm{mm}$

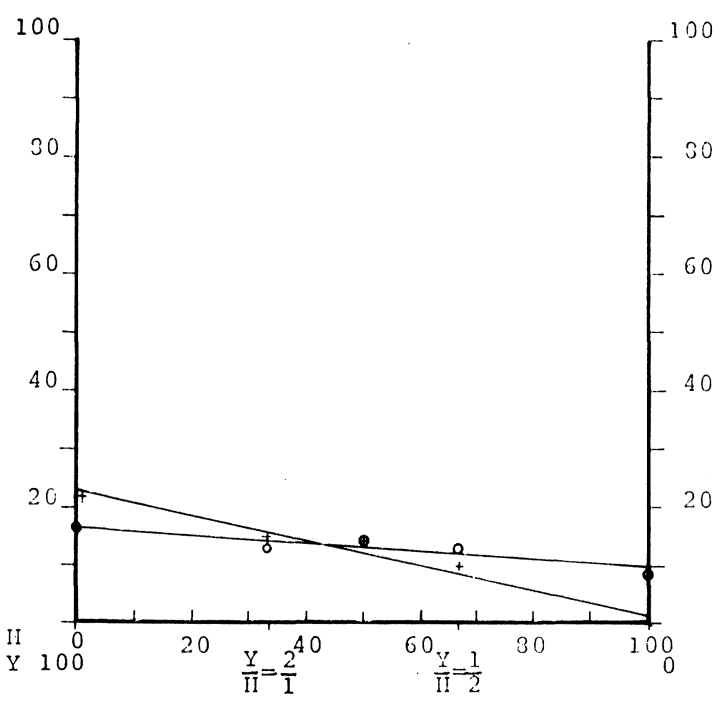

Regresión alturas.

$\mathrm{H}: \mathrm{SO}_{4} \mathrm{Ca} \cdot 1 / 2 \mathrm{H}_{2} \mathrm{O}$.

$Y: \mathrm{SO}_{4} \mathrm{Ca} \cdot 2 \mathrm{H}_{2} \mathrm{O}$.

+ : Alturas picos $130^{\circ} \mathrm{C}\left(h_{1}\right)$

o: Alturas picos $140^{\circ} \mathrm{C}\left(h_{2}\right)$

$\mathrm{SO}_{3}=3,50$ Serie $\mathrm{A}$.

Peso muestra 0,6 $\mathrm{g}$.

Gráfica II

- Regresión alturas.

$\mathrm{H}: \mathrm{SO}_{4} \mathrm{Ca} \cdot 1 / 2 \mathrm{H}_{2} \mathrm{O}$.

$\mathrm{Y}: \mathrm{SO}_{4} \mathrm{Ca} \cdot 2 \mathrm{H}_{2} \mathrm{O}$.

+ : Alturas picos $130^{\circ} \mathrm{C}\left(h_{1}\right)$

o: Alturas picos $140^{\circ} \mathrm{C}\left(h_{2}\right)$
$\mathrm{SO}_{3}=2,75$ Serie $\mathrm{A}$.

P'eso muestra 0,6 g. 
$M \Lambda \Lambda$

" A M N

Mn $\mathrm{M}$

$M M M$

$\sim \Omega \sim$ 
TABLA 2

\begin{tabular}{|c|c|c|c|c|c|c|c|c|c|c|c|c|c|c|c|c|c|c|}
\hline \multirow{2}{*}{$\%$ yeso } & \multicolumn{4}{|c|}{$4,50 \% \mathrm{SO}_{3}$ total } & \multicolumn{2}{|c|}{$\begin{aligned} \text { Serie } & \text { A } \\
\text { Grupo } & \text { la }\end{aligned}$} & \multicolumn{4}{|c|}{$3,50 \% \mathrm{SO}_{3}$ total } & \multicolumn{2}{|c|}{$\begin{aligned} & \text { Serie } \text { A } \\
& \text { Grupo Ila }\end{aligned}$} & \multicolumn{4}{|c|}{$2,75 \% \mathrm{SO}_{3}$ total } & \multicolumn{2}{|c|}{$\begin{array}{c}\text { Serie A } \\
\text { Grupo IIIa }\end{array}$} \\
\hline & $h_{1}$ & $h_{2}$ & $r_{h}$ & $\mathbf{a}_{1}$ & $a_{2}$ & $r_{a}$ & $h_{1}$ & $h_{2}$ & $\mathbf{r}_{\mathrm{h}}$ & $a_{1}$ & $a_{2}$ & $\mathbf{r}_{\mathrm{a}}$ & $\mathbf{h}_{1}$ & $h_{2}$ & $\mathbf{r}_{\mathrm{h}}$ & $a_{1}$ & $a_{2}$ & $\mathbf{r a}_{\mathbf{a}}$ \\
\hline 100 & 100 & 53 & 1,8868 & 1.019 & 514 & 1,9830 & 80 & 44 & 1,8181 & 787 & 305 & 2,1561 & 62 & 33 & 1,8788 & 645 & 335 & 1,9254 \\
\hline 66,6 & 61 & 42 & 1,4524 & 665 & 577 & 1,1525 & 54 & 36 & 1,5000 & 444 & 396 & 1,1212 & 47 & 31 & 1,5161 & 449 & 326 & 1,3773 \\
\hline 50 & 41 & 43 & 0,9535 & 411 & 467 & 0,8801 & 49 & 32 & 1,5312 & 366 & 313 & 1,1693 & 33 & 27 & 1,2222 & 291 & 290 & 1,0034 \\
\hline 33,3 & 28 & 42 & 0,6667 & 360 & 468 & 0,7692 & 33 & 36 & 0,9166 & 270 & 363 & 0,7438 & 21 & 24 & 0,8750 & 199 & 278 & 0,7158 \\
\hline 0 & 0 & 29 & 0 & 0 & 414 & 0 & 0 & 23 & 0 & 0 & 284 & 0 & 0 & 16 & 0 & 0 & 157 & 0 \\
\hline$R_{1}$ & 0,999 & 0,944 & 0,944 & 0,993 & 0,992 & 0,989 & 0,990 & 0,923 & 0,934 & 0,993 & 0,687 & 0,980 & 0,995 & 0,970 & 0,979 & 0,997 & 0,912 & 0,998 \\
\hline
\end{tabular}

\begin{tabular}{|c|c|c|c|c|c|c|}
\hline \multirow{2}{*}{$\%$ yeso } & \multicolumn{4}{|c|}{$4,50 \% \mathrm{SO}_{3}$ total } & \multicolumn{2}{|c|}{$\begin{array}{cc}\text { Serie B } \\
\text { Grupo Ib }\end{array}$} \\
\hline & $\mathbf{h}_{1}$ & $\mathbf{h}_{2}$ & $r_{h}$ & $a_{1}$ & $\mathbf{a}_{2}$ & $\mathbf{r a}_{\mathbf{a}}$ \\
\hline 100 & 93 & 63 & 1,4762 & 1.007 & 558 & 1,8046 \\
\hline 66,6 & 67 & 47 & 1,4256 & 688 & 450 & 1,5289 \\
\hline 50 & 51 & 50 & 1,0200 & 514 & 424 & 1,2122 \\
\hline 33,3 & 40 & 48 & 0,8333 & 374 & 416 & 0,8990 \\
\hline 0 & 0 & 35 & 0 & 0 & 406 & 0 \\
\hline$R_{1}$ & 0,994 & 0,931 & 0,941 & 0,999 & 0,882 & 0,969 \\
\hline
\end{tabular}

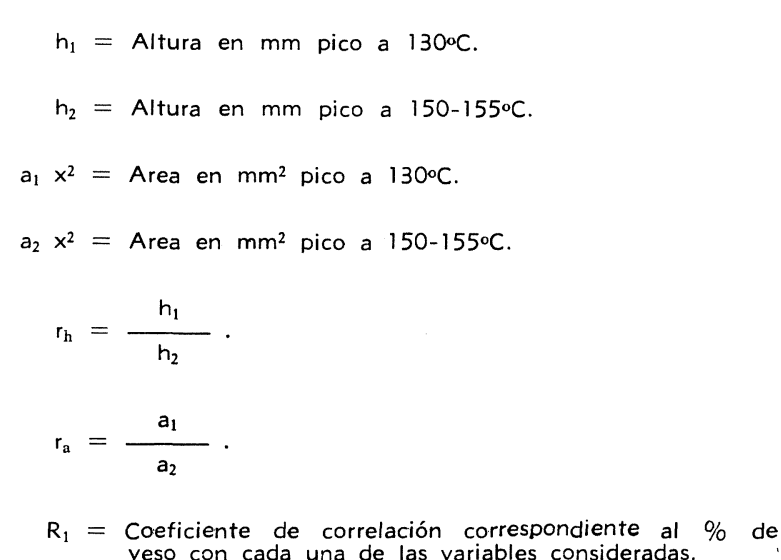

$R_{1}=\begin{aligned} & \text { Coeficiente de correlación correspondiente al } \\ & \text { yeso con cada una de las variables consideradas. }\end{aligned}$ 


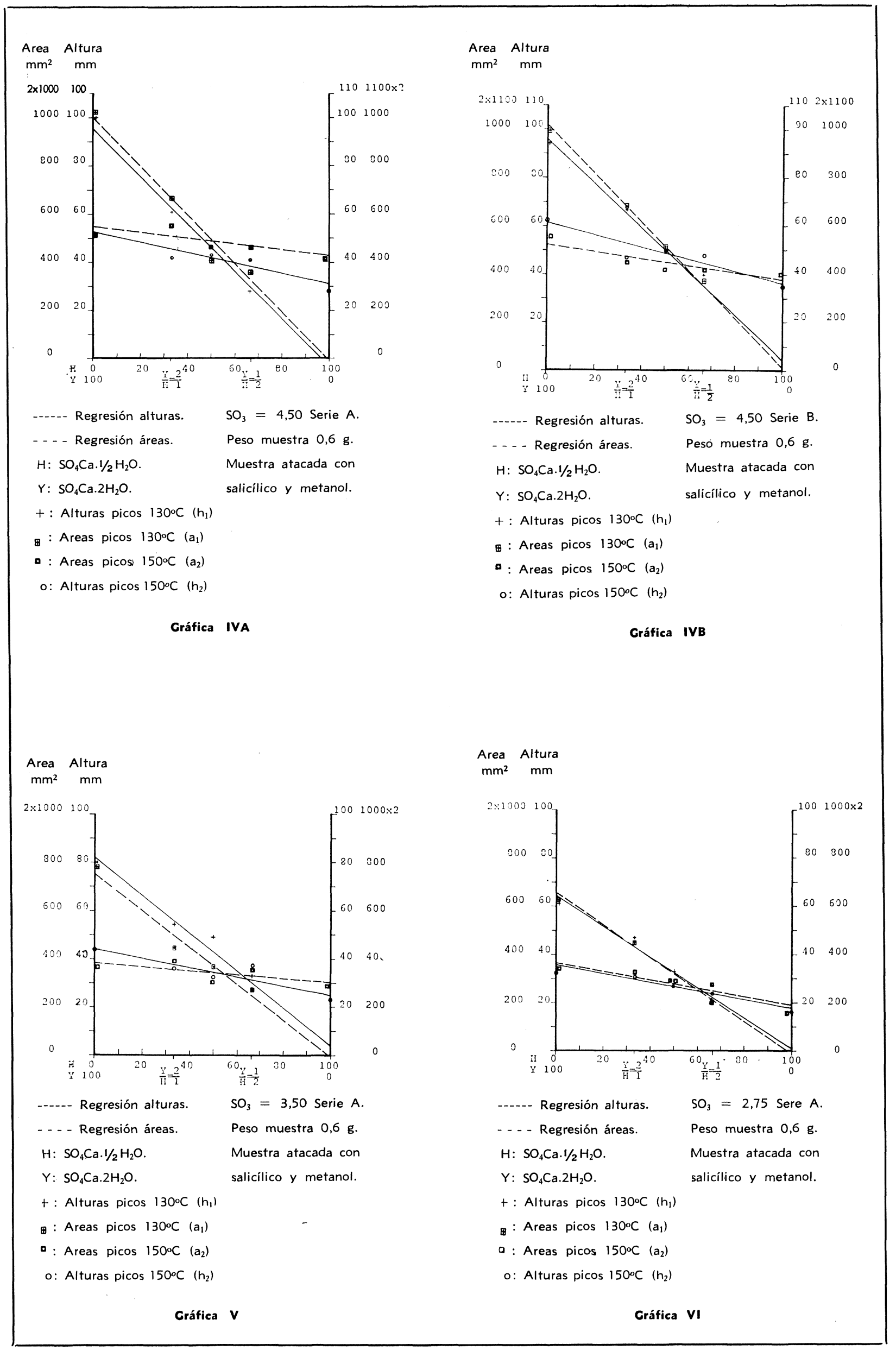




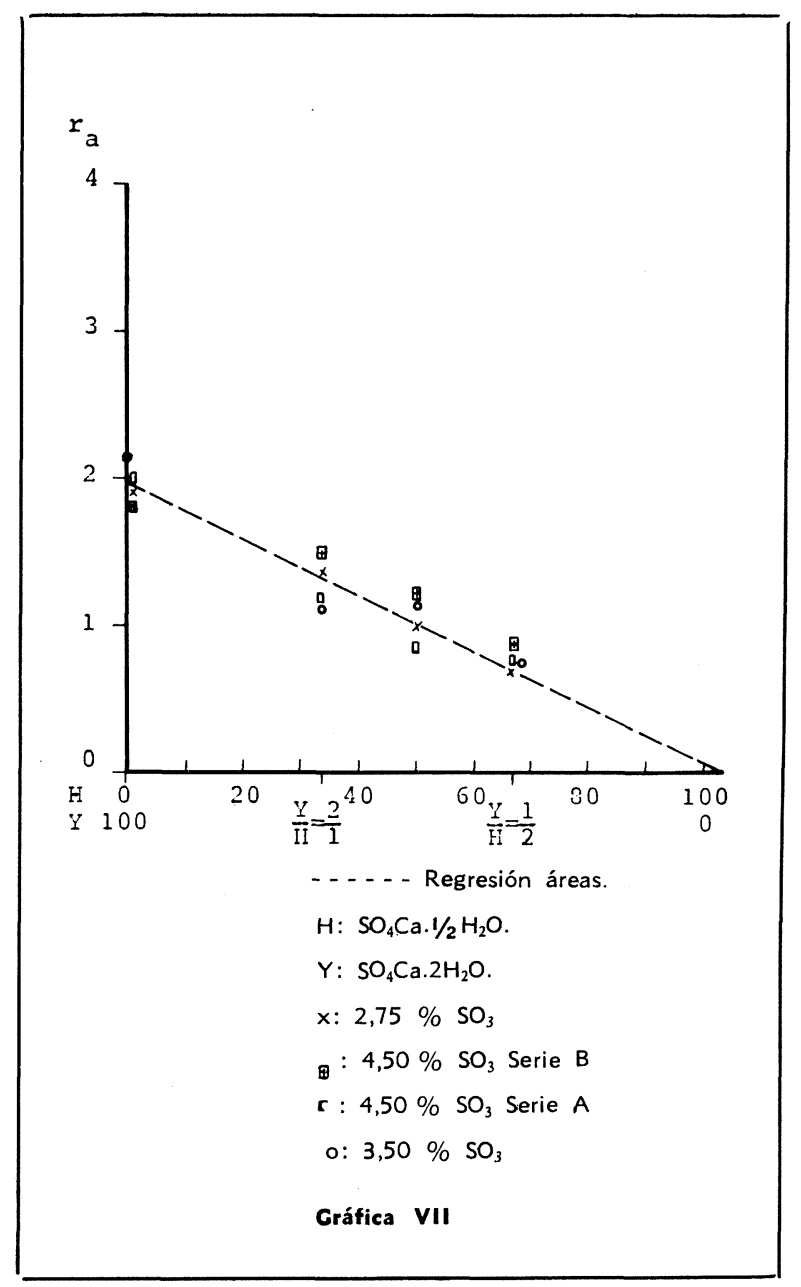

\subsection{Discusión de los resultados}

De los valores obtenidos a partir de los termogramas de las muestras de cemento y de las "concentradas", se deduce:

a) La correlación entre la proporción yesohemihidrato $(\mathrm{Y} / \mathrm{H})$, frente a la altura $\left(\mathrm{h}_{1}\right)$, del pico correspondiente a la pérdida de 1 y $1 / 2$ moléculas de agua de hidratación es muy fuerte. La media de los coeficientes de correlación para las muestras de cemento es de 0,9895 y para las concentradas 0,9945 .

En las muestras concentradas, que son las que constituyen las realmente útiles para el estudio, sólo una tiene una dispersión del $8 \%$; el resto de las muestras es inferior al $5 \%$. Los resultados concuerdan con los citados por otros autores en (5), (6), (7) y (8).

b) La correlación entre la proporción yesohemihidrato $(\mathrm{Y} / \mathrm{H})$ frente a la altura $\left(\mathrm{h}_{2}\right)$ del pico correspondiente a la pérdida de la $1 / 2$ molécula de agua, es más débil que la pérdida de 1 y $1 / 2$ moléculas $\left(\mathrm{h}_{1}\right)$. La media de los coeficientes de correlación para la smuestras de cemento es de 0,942 .

La pendiente de la recta es pequeña y las dispersiones son muy superiores a las obtenidas con las alturas $\mathrm{h}_{1}$; por lo tanto, esta correlación no es la apropiada para emplearla en determinación de la proporción yeso-hemihidrato.

c) En los apartados a) y b) las correlaciones medias obtenidas, al considerar las áreas en lugar de las alturas, son: para las áreas $\left(a_{1}\right)$ del primer pico 0,996 y 0,868 para las áreas $\left(\mathrm{a}_{2}\right)$ del segundo ; este último valor viene muy afectado por el coeficiente de correlación bajo 0,68. Apreciándose como en el caso del apartado b), que la pendiente de la recta de las $a_{2}$ es pequeña y las dispersiones superiores a las de las $a_{1}$.

d) La correlación entre la proporción yeso-hemihidrato frente al cociente $\left(\mathrm{r}_{\mathrm{h}}\right)$, de las alturas del primer pico $\left(h_{1}\right)$, con respecto al segundo $\left(h_{2}\right)$, es fuerte, con una media de los coeficientes de 0,962 . La misma correlación con el cociente de áreas $\left(\mathrm{r}_{\mathrm{a}}\right)$ da un coeficiente medio de 0,984 .

e) Se ha estudiado también la influencia del contenido en $\mathrm{SO}_{3}$ total (entendiendo como tal, la suma de los parciales del yeso, hemihidrato y clínker) en la correlación yesohemihidrato frente a las alturas y áreas de sus respectivos picos. Siendo de interés destacar que la correlación entre yeso-hemihidrato frente al cociente de alturas $\left(r_{h}\right)$ o de áreas $\left(\mathrm{r}_{\mathrm{a}}\right)$ no viene afectada por el contenido de $\mathrm{SO}_{3}$ total en el intervalo considerado $\left(2,75-4,50 \% \mathrm{SO}_{3}\right.$ Gráfica VII); el coeficiente de correlación es de 0,9516 para los $\mathrm{r}_{\mathrm{h}}$ y 0,9808 para $r_{a}$.

Estos resultados concuerdan con lo obtenido por St. John (8), llegando las dispersiones obtenidas hasta el $10 \%$. 
De lo expuesto en estos cinco apartados se deduce, que el método más correcto para determinar la proporción yeso-hemihidrato en un cemento es utilizar las muestras concentradas y emplear la correlación con las alturas $h_{1}$ del primer pico, siempre que el contenido en $\mathrm{SO}_{3}$ del cemento sea igual al de las muestras del tarado, y no tengan influencia otros factores no considerados en el apartado a), como son la finura y naturaleza del yeso y hemihidrato.

El otro método aceptable es emplear la correlación con los $\mathrm{r}_{\mathrm{a}}$, en el que el $\mathrm{SO}_{3}$ del cemento puede variar dentro del intervalo estudiado.

\subsection{Análisis Térmico Diferencial doble (ATD-d)}

Otra técnica de análisis térmico que es posible emplear para la determinación de la proporción yeso-hemihidrato en un cemento, es el ATD-d (análisis térmico diferencial doble).

El ATD-d consiste en sustituir la substancia inerte empleada en el ATD, por una substancia de referencia cuyo análisis térmico es conocido. Como consecuencia se obtiene el termograma correspondiente a la diferencia de los termogramas simples, efectuados simultáneamente, de la muestra-problema y de la referencia.

En nuestro caso, efectuamos el ATD-d sobre las muestras concentradas, utilizando como substancia de referencia la muestra del $100 \%$ de yeso (y). En los termogramas obtenidos fig. 3, se procedió a medir la altura y el área del pico de $130^{\circ} \mathrm{C}$. (Tabla 3 ) y se representaron en la gráfica VIII.

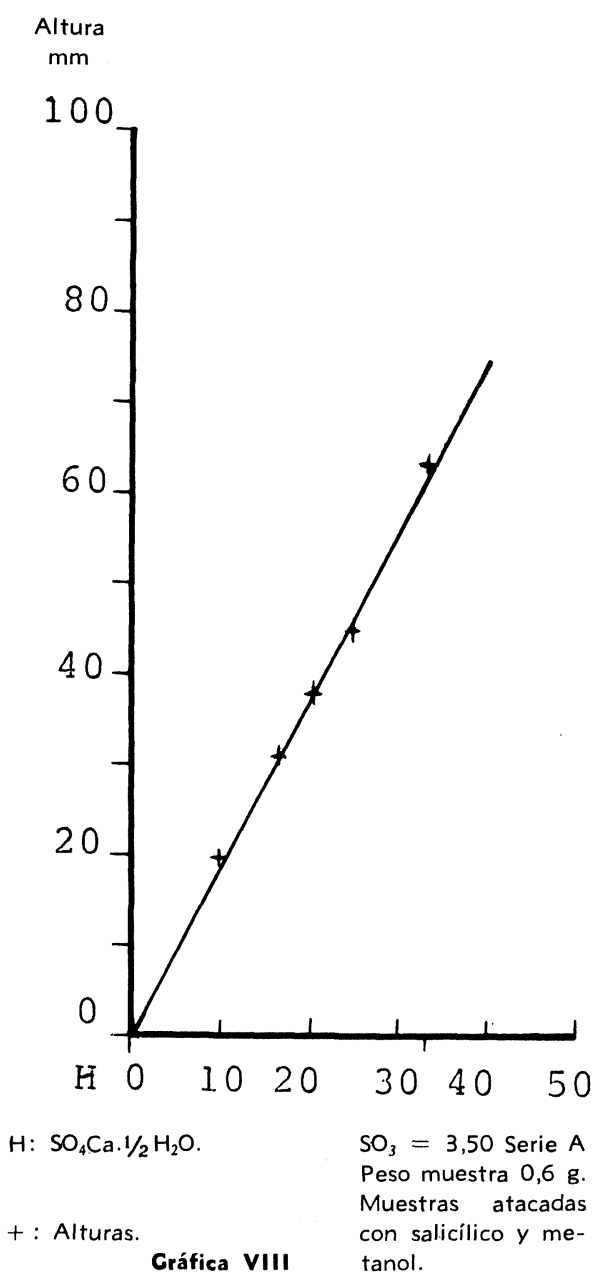

TABLA 3

\begin{tabular}{|c|c|c|c|}
\hline$\%$ yeso & $\%$ hemihidrato & Area ( $\left.\mathbf{m m}^{2}\right)$ & Altura (mm) \\
\hline 100 & 0 & 0 & 0 \\
90 & 10,0 & 166 & 20 \\
83,3 & 16,7 & 252 & 31 \\
79,1 & 20,9 & 250 & 38 \\
75 & 25,0 & 255 & 46 \\
66,6 & 33,3 & 334 & 63 \\
50 & 50,0 & 511 & 71 \\
33,3 & 66,6 & 580 & 76 \\
0 & 100 & 675 & 76 \\
\hline $\begin{array}{l}\text { Coeficiente } \\
\text { correlación }\end{array}$ & & 0,950 & 0,999 \\
\hline
\end{tabular}


Los valores (1) no entraron en el cálculo del coeficiente de correlación, por considerarlos afectados por la asimetría que aparece en los termogramas al incrementar el $\mathrm{H}, \mathrm{o} / \mathrm{y}$ por la pequeña dilución de las muestras también al incrementar el $\mathrm{H}$.

$\%$ yeso $\quad \mathrm{T}^{\circ} \mathrm{C}$

$0 \%$

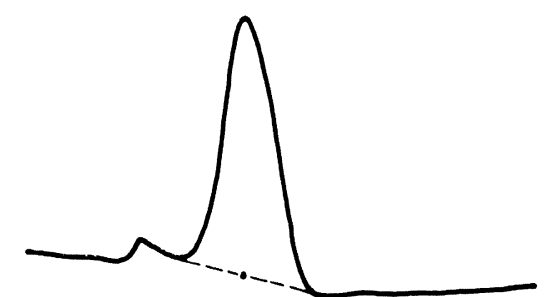

$33,33 \%$

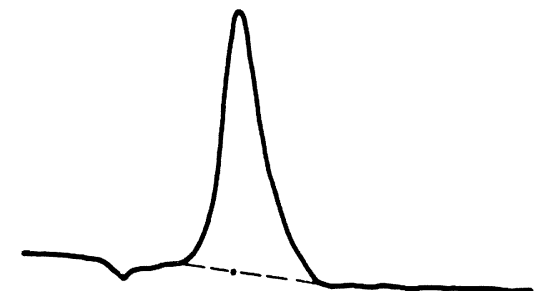

$50,00 \%$

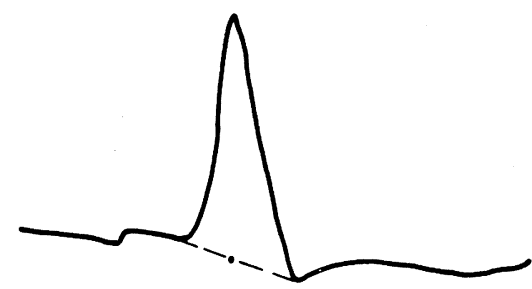

$66,66 \%$

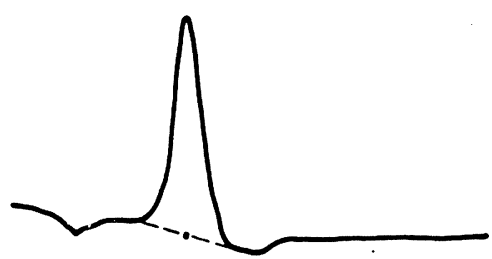

$75,00 \%$

$79,10 \%$

$83,33 \%$

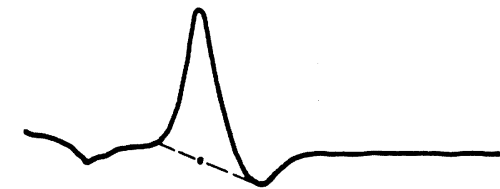

$90,00 \%$
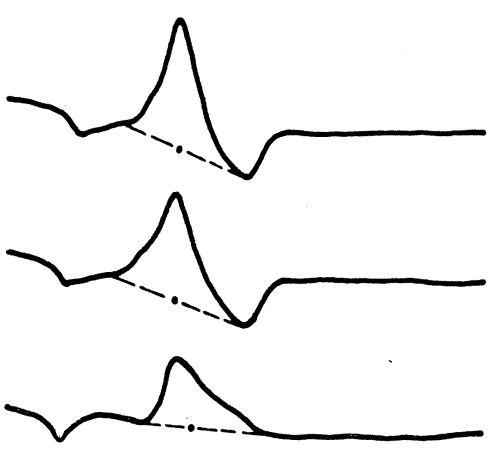

Fig. 3
De lo expuesto se deduce que la correlación entre la proporción de yeso-hemihidrato frente a las alturas del pico a $130^{\circ} \mathrm{C}$ es muy fuerte. Hasta valores de $35 \%$ de hemihidrato, las dispersiones son inferiores al $1 \%$.

Esta precisión es debida a la simultaneidad del análisis de la muestra-problema y de la referencia, lo que implica una igualdad de condiciones para ambas muestras.

Otro aspecto interesante que presenta la técnica del ATD-d, es poder soslayar algunos factores que afectan al ATD, como son: la influencia de la finura del yeso, la naturaleza o génesis del yeso, y los errores en el ataque con salicílico y metanol para concentrar las muestras.

Esto es factible si utilizamos como sustancia de referencia la propia muestra problema con proporción yeso-hemihidrato desconocida, hidratada hasta el $100 \%$ en forma de yeso. Pues en estas circunstancias, la finura y génesis serán las mismas para ambas muestras analizadas.

La dificultad está en la mencionada hidratación desde la proporción desconocida de yesohemihidrato hasta la del $100 \%$ en forma de yeso, sin que las manipulaciones efectuadas con la muestra afecten a la respuesta del análisis térmico. Por este motivo, se realiza posteriormente a la hidratación del hemihidrato un secado con la misión de eliminar la humedad residual de la muestra.

Las condiciones apropiadas parece que, en nuestro caso, son: siete días de hidratación en atmósfera (saturada de humedad) a $20^{\circ} \mathrm{C} \mathrm{y}$, posteriormente, secado a $40^{\circ} \mathrm{C}$ hasta peso constante.

En estas condiciones, la muestra de proporción yeso-hemihidrato del $50 \%$ da termograma idéntico al de la muestra preparada mezclando clínker y yeso.

\section{O N C L U S I O N E S}

De todos los ensayos realizados, se deduce que los resultados obtenidos desde el punto de vista de reproducibilidad y fiabilidad demuestran: que la técnica más idónea a aplicar para la determinación del porcentaje de yeso y hemihidrato en muestras de cemento, 
es la del análisis térmico diferencial doble sobre muestras atacadas previamente con ácido salicílico en solución de alcohol metílico, al menos en aquellas muestras cuyo contenido en hemihidrato no sobrepase el $35 \%$ del total de yeso.

\section{B I B L I O G R A F I A}

(1) Murakami K., Izuka, S. and Tanaka, H.: Sekko To Sekkai, 26, 8 (1957).

(2) Takemoto K., Ito, I., and Hirayama, K.: Rock Products 62, 140, (1959).

(3) Dyczek, J., Sawkow, J. and Sulikowski Jo.: Cement-Wapno-Gips 18, 99 (1963).

(4) Sulikowski y J. Proc.: 7th Conf. Silicate Ind., Budapest p. 137, (1965).

(5) Kuntze, R. A.: Determination of small amounts of gypsum in calcium sulfate hemi hydrate by differential thermal analysis. Mater. Res. Std. 2,640-642 (1962).

(6) Kalousek G. L.: Analyzing $\mathrm{SO}_{3}$-Bearing Phases in Hydrating Cements Mater. Res. Std. 5 (6) 292-304 (1965).

(7) Sato, N., and Kanaya, M.: J. Res. Onada CementCo., 14, 143 (1962).

(8) St. John, D. A.: New Zealand J. Sci, 7,353, (1964).

(9) Dilaktorskir, N. L. and Arkhangel'ska, L. S.: The termal analysis method. Proc. Sth Conf. Exptl. Mineral and Petrogr., Moscow 1956 pp. 88-96 Acad. Sci USSR, Moscow, 1958. 